\title{
La utilización de la tecnología para mejorar la práctica, el entrenamiento y el rendimiento: Resumen práctico
}

\author{
Mark Gellard, Matko Jelcic y Alejandro Vial
}

\section{RESUMEN}

El propósito de este artículo es de resumir la evolución de la ciencia y la tecnología dentro del deporte del tenis, proporcionando anto a jugadores como entrenadores algunas aplicaciones prácticas y específicas que puedan utilizar los jugadores de todas las edades y habilidades.

\author{
Palabras clave: Tecnología, \\ eficiencia en el entrenamiento \\ en pista, Evolución de la \\ ciencia \\ Recibido: 31 Abril 2018 \\ Aceptado: 13 Junio 2018 \\ Autor correspondiente: \\ Mark Gellard. \\ Correo electrónico: \\ mark@firststriketennis.us
}

\section{INTRODUCCIÓN}

En 2006 el tenis experimentó su mayor revolución tecnológica desde que las raquetas de grafito cambiaron el juego en 1980 (Lammer y Kotze, 2003) cuando se introdujo oficialmente el Ojo del Halcón (Hawk-Eye) en el torneo de Miami-Nasdaq 100 de la ATP. Esta revolucionaria tecnología no solamente sentó un precedente tecnológico dentro del deporte sino que también sirvió como herramienta invalorable para los jueces árbitro, los jugadores y los entrenadores, permitiéndoles hacer observaciones con respecto al rendimiento (Boadong, 2014), y básicamente, abriendo las puertas a un aluvión de innovaciones tecnológicas.

La aplicabilidad tecnológica ha ampliado indudablemente sus fronteras para incluir a los deportes en el siglo XXI. Como vemos actualmente, la tecnología reinando con supremacía sobre instituciones como la Agencia Mundial Antidopaje (WADA), la Fédération Internationale de Football Association (FIFA), la International Tennis Federation (ITF), la Women's Tennis Association (WTA) y la Association of Tennis Professionals (ATP), etc. no solo para apoyar a los reglamentos y políticas de sus respectivos deportes (Loland, 2009), sino también para ayudar a mejorar las observaciones y la recopilación de datos (Giblin, Tor, y Parrington, 2016).

El análisis cualitativo es la estrategia que emplean más comúnmente la mayoría de los entrenadores de tenis para identificar y diagnosticar las deficiencias de la estrategia y la técnica, pero ello puede ser problemático debido a las altas velocidades del juego (Elliott y cols., 2003). Además, los especialistas de trabajo en condición física dependen con demasiada frecuencia de la opinión subjetiva con respecto al estado físico, la salud y el rendimiento de un jugador, en lugar de un análisis objetivo que puede, con frecuencia, llevar a sobreentrenamiento, fatiga y lesión. Las obvias desventajas con las técnicas de "análisis subjetivo" mencionadas se han documentado extensamente (Hughes y Franks, 2004), por lo cual, la urgencia por integrar el deporte dentro de la tecnología moderna se ha tornado imperativa. Según Omoregie (2016), para comprender mejor cómo la tecnología ayuda al deporte, es posible dividirla en seis sub-categorías; auto tecnologías, tecnologías de rehabilitación, tecnologías de a información, tecnologías de movimiento, medios tecnológicos y tecnologías de base de datos.

Este estudio intenta ilustrar cómo las tecnologías de base de datos y las de movimiento pueden mejorar ampliamente la efectividad del rendimiento en cancha, tanto del entrenador como del jugador, mejorar el rendimiento físico y la prevención de lesiones fuera de cancha, y simultáneamente, proporcionar algunos dispositivos de entrenamiento específicos, al tiempo que advertimos a los usuarios sobre los riesgos potenciales asociados con la abundancia de información actual a nuestra disposición. 


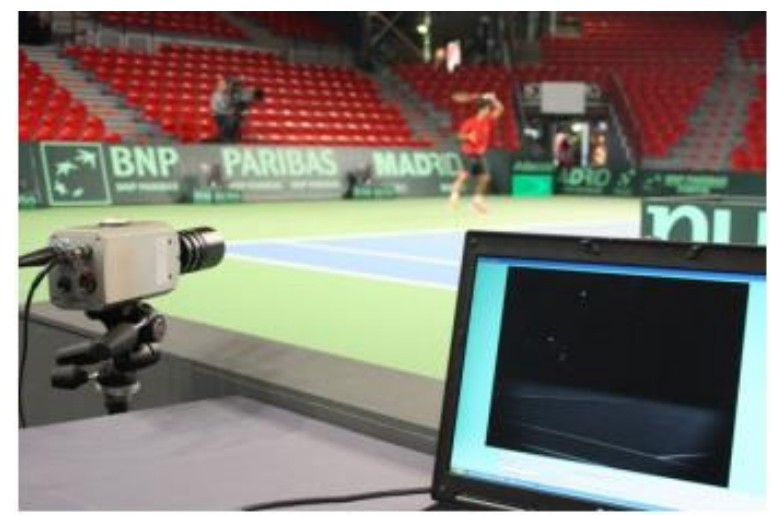

TECNOLOGÍA "EN CANCHA "

Por lo que a la cancha se refiere, los entrenadores se focalizan principalmente en dos componentes clave del desarrollo: maximizar la eficiencia técnica (Schönborn, 2000), de manera que los golpes puedan ejecutarse repetidamente, e implementar una base estratégica correcta que maximice las capacidades mentales y físicas del individuo. El $75 \%$ de toda la información procesada por el cerebro está en formato visual (Williams, 2009), por lo que la llegada de las aplicaciones de análisis por video en cámara lenta que están disponibles en dispositivos móviles, están aayudando mucho tanto a jugadores como a entrenadores. Además, según demuestran muchos estudios (Jones y Stuth, 1997) el uso de las imágenes mentales (fotos, video, etc.), combinado con la práctica física, pueden mejorar significativamente la eficiencia de la acción motriz que se está adquiriendo.

Las aplicaciones prácticas, económicas y de uso fácil, como 'Coaches' Eye', 'HUDL Technique' y 'CoachMyVideo' se están haciendo más popular en el mundo del tenis, ofreciendo gran cantidad de funciones entre las que se incluyen "la comparación en pantalla dividida", "la vista transparente/ lado a lado" y otras funcionalidades más comunes como los "medidores de tiempo y cronómetro", las "herramientas de medición", los "ángulos", el "zoom", la "imagen espejo", las "capturas de pantalla" y la "visualización de secuencia de imágenes compuesta" (secuencia de fotos). Otras opciones más robustas como Dartfish y Siliconcoach son desde hace tiempo consideradas como el moedlo de referencia del video análisis, pues proporcionan al usuario una cantidad de funciones adicionales como "video" de alta definición y "etiquetado" de partidos, lo cual permite ver partidos enteros mientras se agrupan simultáneamente puntos en distintas categorías específicas como "golpes ganadores con la derecha", "errores con el revés", o "saques abiertos directos", se puede enumerar y averiguar las acciones y los patrones de juego tanto del jugador como de su adversario. Pero, tales programas son considerablemente más caros y consumen mayor tiempo de uso que las aplicaciones móviles mencionadas anteriormente, lo que las vuelve menos prácticas en el mercado actual.
Para intentar mejorar la comprensión del juego de los jugadores, implementar patrones de juego específicos o incrementar la consciencia estratégica general, los entrenadores se basan cada vez más en el análisis estadístico y en la compilación de datos. Sorprendentemente, $90 \%$ de los datos mundiales se ha generado en sólo los dos últimos años (ScienceDaily, 2013); el tenis ha demostrado recientemente su compromiso con la modernización tecnológica; el circuito WTA acaba de asociarse con SAP que le proporcionará estadísticas en tiempo real y mediciones de datos durante los partidos para ayudar a los entrenadores con la planificación y las estrategias del juego. Además, la ITF ha implementado estadísticas detalladas para todos los partidos del cuadro principal de sus torneos del circuito profesional, que están disponibles (en tiempo real) por medio de la aplicación "Circuito Profesional de la ITF" (iOS y Android) permitiendo a mucho jugadores "de transición" la oportunidad única de lograr una visión sobre su propio rendimiento.

Con más interés para las estadísticas y los datos, se han hecho disponibles muchas más aplicaciones para el "seguimiento de partidos", como Tennis Stats HD, Pro Tennis Tracker, Tennis Trakker, Tennis Math, TennisStats y SmashPoint, las cuales todas proporcionan una gran cantidad de información, como los errores no forzados, los tiros ganadores, el \% de servicios, y los puntos de rotura salvados, además de una cantidad de información relevante que se ha tornado muy apreciada y frecuente en los deportes profesionales (Haigh, 2009). Estas aplicaciones están disponibles por un pago nominal, y ayudan a los entrenadores a evaluar objetivamente el rendimiento que, como sugieren los estudios, es esencial (Franks y Miller, 1991). La habilidad de un entrenador de recordar un partido con precisión tras su finalización es relativamente baja (inferior al $40 \%)$, por lo tanto, la necesidad de registrar los datos ha crecido. Craig O'Shannessy (2014) se ha transformado en un pionero del análisis del tenis, de la recopilación de datos y de la implementación de estrategias, poniendo de manifiesto, por ejemplo, la importancia de los primeros golpes de cada punto, indicando que la duración más frecuente en el tenis profesional es de sólo 1 tiro

La retroalimentación cualitativa se está haciendo más redundante a medida que el análisis cuantitativo se hace más accesible, proporcionando hechos no sesgados y objetivos, en oposición a la opinión subjetiva. Play Sight se ha transformado en un sinónimo de la tecnología del tenis moderno, pues su sistema cámara-y-kiosco transforma la cancha de tenis tradicional en una maravilla tecnológica, por medio de los kioscos con pantalla táctil interactiva, las cámaras de alta definición, el procesamiento avanzado de imágenes, y los algoritmos analíticos únicos que proporcionan a los jugadores una completa evaluación de sus entrenamientos / partidos, detallan objetivamente las áreas clave como el tipo de golpe, la trayectoria, la velocidad y el efecto de la pelota y el movimiento del jugador, hasta puede ofrecer cantar las líneas, 
realizar la transmisión de video en tiempo real y las repeticiones de video desde múltiples ángulos.

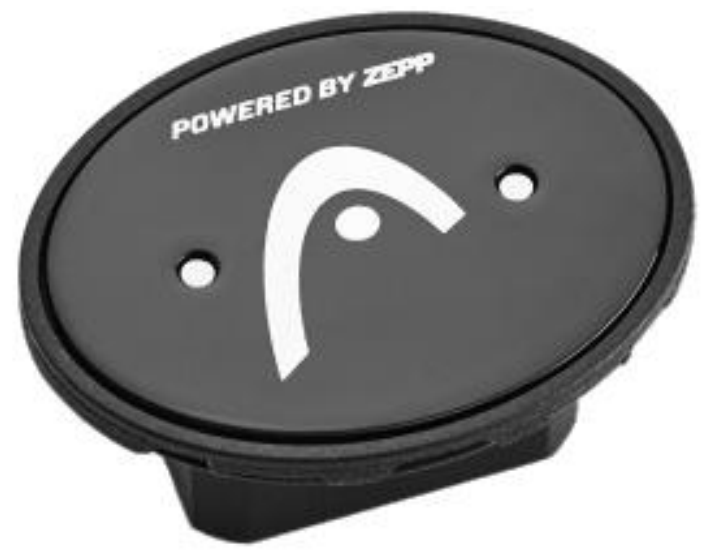

Además, algunas compañías como Babolat, Head, Zepp, Yonex, Sony y Wilson están ahora explotando la tecnología moderna brindando a los jugadores la oportunidad de convertir sus raquetas de tenis en una "máquina analítica", por medio del uso de sensores para raquetas que se adhieren fácilmente a éstas. Según Daniel Becker, gerente de marketing senior de Babolat, el sensor incorporado utiliza un "acelerómetro que analiza la dirección de la raqueta y un giroscopio que analiza la rotación de la raqueta" además de un sensor "piezoeléctrico que analiza la vibración para informar la ubicación de la pelota en la raqueta" (marketwatch.com, 2015).

\section{TECNOLOGÍA "FUERA DE CANCHA"}

Cuando los preparadores físicos tratan de mejorar la condición físical de sus deportistas y evitar las lesiones, buscan lograr una ventaja competitiva a través del entrenamiento de la fuerza y el acondicionamiento físico. Desde el punto de vista de la evaluación del deportista, una herramienta útil es Omegawave; un pequeño dispositivo portátil para evaluar las adaptaciones a corto y largo plazo, que ocurren en el cuerpo humano. El dispositivo cporta información pertinente como la actividad cardíaca (ECG), la actividad de las ondas cerebrales ultra lentas, la fatiga neuromuscular, y la velocidad de reacción, las cuales se pueden ver y analizar al instante. Monitoreando los cambios específicos, los preparadores físicos pueden modificar los protocolos de entrenamiento, puesto que el sistema Omegawave proporciona información aplicable que puede ayudar al deportista para mejorar su resistencia al estrés, y la capacidad de trabajo, evitar el sobre entrenamiento y reducir el riesgo de lesiones (Fomin, Nasedkin, 2013). Todas las mediciones son almacenadas en un sistema basado en la nube que proporciona los resultados y las recomendaciones pertinentes para los sistemas cardíaco, metabólico, sistema nervioso central y hormonal, todos marcadores primarios a la hora de determinar si un deportista está en condiciones aptas para una actuación exitosa en un momento dado de la temporada.

Desde el punto de vista del monitoreo del deportista, el Velocity Based Training (VBT) se ha convertido en el principal método para determinar la carga del entrenamiento de la fuerza. El 'Push Band' e un sistema que mide la velocidad del movimiento por medio de un acelerómetro 3D y un Giroscopio que permite que los preparadores monitoreen de forma inmediata la fatiga y la preparación por medio de la identificación de las reducciones de velocidad de movimiento y de la potencia. La potencia suele ser dejada de lado por parte de los preparadores físicos y los atletas, pero la fuerza es el único factor de la ecuación $(F=m a)$, (Zatsiorsky, 1995), diferentes fuerzas tienen diferentes velocidades (Verkhoshansky, 1982), entonces, este dispositivo portátil asegura que se vea el desarrollo adecuado a través de todo el continuo fuerza- velocidad. Varios factores, como el estado actual del entrenamiento, el modelo de periodización elegido, la potencia, la velocidad y las repeticiones promedio máximas son explicadas automáticamente por este dispositivo, y la información generada ayuda a los entrenadores a determinar la carga óptima sin depender de la simple vista o del nivel del esfuerzo percibido.

\section{CONCLUSIONES}

Cada uno de estos avances tecnológicos proporciona a entrenadores, jugadores, padres y preparadores información útil que modificará significativamente los protocolos, las evaluaciones de los partidos, el programa de torneos, la prevención de lesiones y más. Sin embargo, los peligros son evidentes, pues ahora más que nunca los "educadores" deben asegurarse de que sus consejos sean sólidos, la información que proporcionen sea objetiva, y la interpretación de los datos obtenidos sea eraz, pues en el siglo 21 "todos" pueden fácilmente acceder a información detallada y a estadísticas asumiendo el rol de "experto".

Ya sea utilizando el análisis por video, los procesadores de salida de potencia, u otro aparato similar, es importante hacer uso de la tecnología de manera que sea beneficiosa para todos los miembros de la industria del deporte. La adquisición y procesamiento de datos/mediciones mejorados, las mejores observaciones y pruebas, el mejor equipamiento y las formas de ayuda para el entrenamiento, por sí solos, no son suficiente retroalimentación para mejorar substancialmente el rendimiento (Giblin, Tor, y Parrington, 2016). Una interpretación informada de los datos proporcionados por la tecnología será, en última instancia, uno de los aspectos más importantes del proceso, a medida que buscamos entornos de práctica más eficientes (Liebermann, y cols. 2002). La revolución tecnológica actual proporciona una multitud de nuevas opciones, pero a la vez, crea gran cantidad de peligros. La tecnología y el entrenamiento solamente son efectivos cuando existe una cultura bien definida y bien establecida, cuando coexisten las personas disciplinadas con el pensamiento disciplinado. Después de todo, esto no es más que el inicio.

\section{REFERENCIAS}

Baodong, Y. (2014). Hawkeye technology using tennis match. Computer Modelling \& New Technologies, 18 (12C), 400-402. 
Elliott, B., Reid, M., Crespo, M. (2003). Biomechanics of Advanced Tennis. London: International Tennis Federation

Fomin R.N., Nasedkin, V. V. (2013). Effective Management of Athlete Preparation: A Comprehensive Approach to Monitoring of Athlete's Individual Readiness. White Paper, Omegawave epub

Franks, I \& Miller, G. (1991). Training Coaches to Observe and Remember. Journal of Sports Sciences, 9 (3), 285-297. https://doi.org/10.1080/02640419108729890

Giblin, G., Tor, E., \& Parrington, L. (2016). The impact of technology on elite sports performance. Sensoria: A Journal of Mind, Brain \& Culture. https://doi.org/10.7790/sa.v12i2.436

Haigh, J., 2009. Uses and limitations of mathematics in sport. IMA Journal Management Mathematics 20(2), 97-108. https://doi.org/10.1093/imaman/dpn024

Hughes, M.D. and Franks, I.M. (2004). Notational Analysis of Sport 2nd Edition - better systems for improving coaching and performance. London: E. \& F.N. Spon.

Jones, L., \& Stuth, G. (1997). The Uses of Mental Imagery in Athletics: An Overview. Applied and Preventive Psychology, 6, 101-115. Retrieved. https://doi.org/10.1016/S0962-1849(05)80016-2

Kutz, S. (2015, September 4). What it's like to play tennis with a 'smart' racket that sends you data. Market Watch. Retrieved from https://www.marketwatch.com/story/what-its-like-toplay-witha- smart-tennis-racket-2015-09-03

Lammer, H., Kotze, J. (2003). Materials and tennis rackets. Mater. Sport. Equipment, Woodhead Publishing, 1, 222-248. https://doi.org/10.1533/9781855738546.2.222

Liebermann, D., Katz, L., Hughes, M., Bartlett, R., McClements, J., \& Franks, I. (2002). Advances in the application of information technology to sport performance. Journal of Sport Sciences, 20, 755-769. https://doi.org/10.1080/026404102320675611

Loland, S., (2009). The ethics of performance-enhancing technology in sport. Journal of the philosophy of sport, 36, 152-16i. https://doi.org/10.1080/00948705.2009.9714754

Omoregie, P. O. (2016). The Impact of technology on sport performance. Proceedings of INCEDI 2016 Conference 29th31st August 2016, Accra, Ghana.

O'Shannessy, C. Brain Game Tennis (2014).The First 4 shots, the mode $=1$. Retrieved from https://www.braingametennis.com/ the-first- -4-shots/the-mode-1/
Schönborn, R. (2000). Advanced Techniques for Competitive Tennis (2nd Ed.). Aachen: Meyer \& Meyer Sport

SINTEF. (2013, May 22). Big Data, for better or worse: $90 \%$ of world's data generated over last two years. ScienceDaily. Retrieved May 23, 2018 from www.sciencedaily.com/releases/2013/05/130522085217.htm

Verkhoshansky V., (1982). The Fundamentals of Special-Strengths Training, Sportivny Press

Williams, R, (2009). Visual Learning Theory. http://www. aweoregon.org/research_theory.html

Zatsiorsky, V.M., (1995). Science and Practice of Strength Training. Champaign, IL. Human Kinetics.

CONTENIDO ITF ACADEMY RECOMENDADO (HAZ CLICK ABAJO)

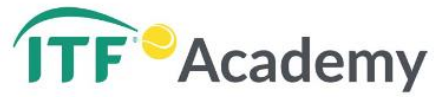

Derechos de Autor (c) 2018 Mark Gellard, Matko Jelcic y Alejandro Vial.

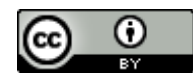

Este texto está protegido por una licencia CreativeCommons 4.0 .

Usted es libre para Compartir — copiar y redistribuir el material en cualquier medio o formato- y Adaptar el documento - remezclar, transformar y crear a partir del material- para cualquier propósito, incluso para fines comerciales, siempre que cumpla la condición de:

Atribución: Usted debe dar crédito a la obra original de manera adecuada, proporcionar un enlace a la licencia, e indicar si se han realizado cambios. Puede hacerlo en cualquier forma razonable, pero no de forma tal que sugiera que tiene el apoyo del licenciante o lo recibe por el uso que hace de la obra.

Resumendelicencia - Textocompletodelalicencia 